\begin{tabular}{|c|l|}
\hline Title & $\begin{array}{l}\text { Both antiviral activity and intracellular localization of chicken Mx protein depend on a polymorphism at amino acid } \\
\text { position 631 }\end{array}$ \\
\hline Author(s) & Sasaki, Keisuke; Yoneda, A kihiro; Ninomiya, A kinori; Kawahara, Manabu; W atanabe, Tomomasa \\
\hline Citation & $\begin{array}{l}\text { Biochemical and Biophysical Research Communications, 430(1), 161-166 } \\
\text { https://doi.org/10.1016/.bbrc.2012.11.053 }\end{array}$ \\
\hline Issue Date & 2013-01-04 \\
\hline Doc URL & http://hdl.handle.net/2115/52138 \\
\hline Type & article (author version) \\
\hline File Information & BBRC430-1_161-166.pdf \\
\hline
\end{tabular}

Instructions for use 


\section{Both antiviral activity and intracellular localization of chicken Mx protein depend on a polymorphism at amino acid position 631}

Keisuke Sasaki, Akihiro Yoneda, Akinori Ninomiya, Manabu Kawahara, Tomomasa Watanabe

Graduate School of Agriculture, Hokkaido University, Sapporo 060-8589, Japan

Corresponding author:

Dr. Manabu Kawahara, Ph.D.

Laboratory of Animal Breeding and Reproduction, Graduate School of Agriculture,

Hokkaido University, Sapporo 060-8589, Japan.

Tel. \& Fax: +81117062541

E-mail address: k-hara@anim.agr.hokudai.ac.jp 


\section{ABSTRACT}

The Mx protein is known to inhibit the multiplication of several RNA viruses. In chickens, a polymorphism at amino acid position 631 (631 aa) of Mx protein has been suggested to be involved in the antiviral ability against vesicular stomatitis virus (VSV) and influenza virus, indicating that a Ser-to-Asn substitution at 631 aa is the source of this antiviral ability. However, how the substitution at 631 aa contributes to the antiviral activity remains to be clarified. In this study, we investigated differences in antiviral activity against VSV and intracellular localization between Ser and Asn types at 631 aa of the chicken Mx protein. The results showed that chicken Mx protein with an Asn at 631 aa inhibited VSV multiplication and Mx distribution in a granular-like pattern in the cytoplasm. However, Mx carrying the Ser type did not inhibit viral growth and homogenous spread throughout the cytoplasm. Furthermore, we found that replacing Ser with Asn at 631 aa provided Mx with antiviral activity against VSV, with Mx showing granular-like distribution in the cytoplasm. These results demonstrate that a single amino acid polymorphism at 631 aa of the chicken Mx protein altered both the antiviral activity and intracellular localization. 


\section{Keywords}

Mx, Chicken, Polymorphism, Vesicular stomatitis virus, Intracellular localization

\section{Abbreviations}

Mx, myxovirus (influenza virus) resistance 1, interferon-inducible protein p78 (mouse);

VSV, vesicular stomatitis virus; leucine zipper, LZ.

\section{Introduction}

Mx protein, a product of an interferon-stimulated gene, is a dynamin-like GTPase,

known to be involved in inhibiting the multiplication of several RNA viruses, including

Orthomyxoviridae, Rhabdoviridae, Paramyxoviridae, Bunyaviridae and Togaviridae as

well as some DNA viruses, including Hepadnaviridae [5,7,10,11,13,15,22]. Generally,

Mx proteins are composed of 4 essential conserved domains, including a GTP-binding 
domain (GD) comprised of 3 sections near the N-terminus, self-assembly sequences (SAS), central interactive domain (CID), and a GTPase effector domain (GED) attached to the C-terminus. The GED contains 2 leucine zippers (LZs), LZ1 and LZ2 [4,14,18]. In humans, the MxA protein possesses the antiviral activity against influenza virus and vesicular stomatitis virus (VSV), with a granular-like distribution in the cytoplasm because of the formation of homo-oligomers that consist of 20-30 molecules [6]. It has also been suggested that both CID and GED bind during viral invasion, leading to a conformational change in the Mx proteins $[6,14,16]$. However, it is not clear whether the intracellular localization of Mx proteins is correlated with its antiviral potential.

It has been demonstrated that a polymorphism at position 631 amino acid (631 aa) of chicken Mx protein plays an important role in the antiviral activity as well as for different survival rates of chicken against H5N1 influenza virus and VSV infections, as previously described $[3,12,19]$. Our research indicated that the antiviral activity of the genotype corresponding to Asn at 631 aa is higher than that of Ser in in vitro experiments using the mouse 3T3 cell line [12]. Furthermore, other in vivo studies have suggested that chicken Mx proteins with an Asn type at 631 aa show the higher 
pathogenic ability against the H7N1 influenza virus than the Ser type and also exhibits more effective inhibition of the H5N2 influenza virus multiplication $[3,19]$. However, several studies have suggested that the 631 aa position of the chicken Mx protein does not influence antiviral activity according to the results of in vitro and in vivo infectious experiments performed using other cultured cell types and other virus strains [1,17]. It remains to be clarified whether the polymorphisms at 631 aa of chicken Mx proteins affect their antiviral activity.

In mice, the MX1 protein is known to localize in the nucleus and inhibit multiplication of influenza virus growing in the host cell nuclei [24]. In contrast, mouse MX2 protein localizes to the cytoplasm and inhibits the multiplication of VSV in the cytoplasm, but not the influenza virus [23]. Thus, it has been suggested that the antiviral activity and intracellular localization of $\mathrm{Mx}$ proteins correlate with compartmentalization in which each virus multiplies in host cells. Only one report states that chicken $\mathrm{Mx}$ is a cytoplasmic protein; however, the relationship between the antiviral activity and intracellular localization has not been directly examined [2]. We hypothesized that the intracytoplasmic localization of chicken Mx protein is associated with its antiviral 
activity toward some viruses.

\section{Materials and Methods}

\subsection{Chickens}

Peripheral blood obtained from 3 chicken breeds, Nagoya (NG), Rhode Island Red (RI), and Shamo (SHK), were collected for total RNA extraction from blood mononuclear cells. NG and SHK are Japanese native breeds, and they were provided by the Hokkaido Livestock Experiment Station (Japan) as previously described [12]. RI was provided by the Field Science Center for Northern Biosphere, Hokkaido University, Japan. 


\subsection{Cell culture}

After collecting chicken peripheral blood, mononuclear cells were isolated using HISTOPAQUE-1077 (Sigma-Aldrich, St. Louis, MO). Next, the cells were cultured in RPMI-1640 medium (Sigma-Aldrich) containing 10\% fetal bovine serum (ICN Bio-Source International, Camarillo, CA), $1.0 \mu \mathrm{L} / \mathrm{mL}$ phytohemagglutinin (PHA-P, Sigma-Aldrich), $\quad 10 \mu \mathrm{L} / \mathrm{mL}$ lectin from Phytolacca Americana (pokeweed) (Sigma-Aldrich), $100 \mathrm{U} / \mathrm{mL}$ penicillin (Nacalai Tesque, Japan), and $100 \mu \mathrm{g} / \mathrm{mL}$ streptomycin (Nacalai Tesque) at $37^{\circ} \mathrm{C}$ under $5 \% \mathrm{CO}_{2}$ for $42 \mathrm{~h}$. Subsequently, to induce Mx mRNA transcription, we added $1000 \mathrm{U} / \mathrm{mL}$ INTRON-A (Schering-Plough, Kenilworth, NJ) to the culture medium and incubated the mononuclear cells for an additional $6 \mathrm{~h}$, as previously described [12].

Mouse BALB/c 3 T3 cultured cells (RCB0163) carrying non-functional Mx genes were purchased from Riken Bioresource Center Cell Bank (Japan) and cultured in Dulbecco's modified Eagle's medium (DMEM; Sigma-Aldrich) containing 10\% fetal bovine serum, $100 \mathrm{U} / \mathrm{mL}$ penicillin, and $100 \mu \mathrm{g} / \mathrm{mL}$ streptomycin at $37^{\circ} \mathrm{C}$ under $5 \% \mathrm{CO}_{2}[9,10] .3 \mathrm{~T} 3$ 
cells were passaged using $0.25 \%$ trypsin (Nacalai Tesque) and $0.02 \% 2 \mathrm{NA}(\mathrm{EDTA} \cdot 2 \mathrm{Na})$

before they attained confluence.

\subsection{Cloning and sequencing of chicken $M x c D N A$}

Total RNAs from mononuclear cells treated with INTRON-A were extracted using

ISOGEN (Nippon Gene, Japan) and were reverse-transcribed using ReverTra-Plus(TOYOBO, Japan) to synthesize cDNA. PCR was performed using the ASTEC Program Temp Control System Pc-700 (ASTEC, Japan). Mx cDNA was amplified using GoTaq DNA polymerase (Promega, Madison, WI) in $5 \mathrm{mM}$ each of the forward and reverse primers for cMx $141 \mathrm{~F}$ (5'-ATGAACAATCCATGGTCCAAC-3') and cMx 2258 R (5'-AGATCTCTACAGGACTTAAAGTCTACC-3'), and $1.0 \mathrm{mM}$ of each dNTP. The cycling profile included an initial denaturing step for $5 \mathrm{~min}$ at $95^{\circ} \mathrm{C}$, followed by 35 cycles at $95^{\circ} \mathrm{C}$ for $0.5 \mathrm{~min}, 55^{\circ} \mathrm{C}$ for $1 \mathrm{~min}, 72^{\circ} \mathrm{C}$ for $2.5 \mathrm{~min}$, and a final extension at $72^{\circ} \mathrm{C}$ for $2.5 \mathrm{~min}$. Next, $M x$ cDNA amplicons were cloned into the pGEM-T Easy Vector (Promega) by using T4 ligase (Promega). Nucleotide sequences of the chicken 
$M x$ cDNA construct within the plasmid DNA were confirmed using a DNA Sequencer

LI-COR Model 4000 (ALOKA, St. Lincoln, NE) and ABI PRISM 310 Genetic Analyzer (PerkinElmer Inc., Waltham, MA). Nucleotide sequences were compared to the NCBI database (GenBank ID: NM 204609), which records the chicken $M x$ mRNA sequence of a White Leghorn breed (WLR).

\subsection{Construction of chicken Mx mRNA expression cells for infectious experiment}

To evaluate the antiviral activity of the chicken Mx protein, each $M x$ cDNA construct derived from the 3 breeds - NG, RI, and SHK breeds - was subcloned into the NotI site of the pCI-neo vector (pCI-neo Mammalian Expression Vector; Promega). These cloning procedures were carried out as described in section 2.3. Plasmid DNAs were purified using the PureYield Plasmid Midiprep System (Promega). Next, using the FuGENE6 transfection reagent (Roche Diagnostics, Switzerland), 200 ng of each chicken $M x$ cDNA construct within the pCI-neo vector was transfected into 3T3 cells. Transfected cells were selectively cultured by the addition of G418 disulfate (Nacalai 
Tesque). After treatment with $0.25 \%$ trypsin and $0.02 \% 2 \mathrm{NA}(\mathrm{EDTA} \cdot 2 \mathrm{Na})$, a single transfected cell showing tolerance to G418 disulfate was cultured for an additional 2 weeks. $M x$ mRNA expression in the cloned cells was confirmed using RT-PCR (data not shown). Cloned cells expressing chicken $M x$ mRNA were used to measure antiviral activity.

\subsection{VSV $\Delta G^{*}-G$ infectious experiment}

As previously described [12], infection experiments were performed using recombinant VSV (VSV $\Delta \mathrm{G}^{*}-\mathrm{G}$ ) carrying the Gfp gene rather than the VSIVgp4 gene coding a glycoprotein ( $\mathrm{G}$ protein) precursor, which was provided by Prof. A. Takada (Research Center for Zoonosis Control, Hokkaido University, Japan) [21]. Cloned cells expressing chicken $M x$ mRNA were passaged into antibiotic-free DMEM and subjected to the infection experiments with $\mathrm{VSV} \Delta \mathrm{G}^{*}$-G. After an additional $12 \mathrm{~h}$ of incubation, infected cells showing green fluorescent protein (GFP)-fluorescence were observed under UV irradiation. Relative infectivity of each cloned cells against VSV $\Delta \mathrm{G}^{*}-\mathrm{G}$ was 
determined by calculating the proportion of GFP fluorescence-expressing cells versus all cultured cells in 10-20 microscope fields. Five independent experiments were carried out for each cloned cell. As negative controls, we examined the relative infectivity in non-transfected 3T3 cells (3T3) and empty pCI-neo vector-transfected 3T3 cells (pCI-neo).

\subsection{Intracellular localization of GFP-chicken Mx fused protein}

To determine intracellular localization of chicken Mx protein, we used a GFP-chicken Mx-fused protein expression vector. First, chicken $M x$ cDNA constructs were subcloned from the pGEM-T Easy vector into the pEGFP-C3 vector (Clontech, Palo Alto, CA), by utilizing the XhoI and $X m a I$ sites. These processes were conducted as mentioned in section 2.3; the plasmid DNAs obtained were purified using the PureYield Plasmid Midiprep System.

3 T3 cells were transfected with chicken $M x$ cDNA constructs in the pEGFP-C3 vector. Twenty-four hours after transfection of each construct, GFP-fluorescent signals 
observed from individual cells were detected under an ultraviolet microscope. Five independent experiments were conducted for each type of cell, and empty vector-transfected cells were used as negative controls (GFP).

\subsection{Construction of artificially mutated chicken Mx cDNA}

To examine the effect of a single amino acid substitution with respect to both antiviral activity and intracellular localization more precisely, we constructed 2 types of artificially mutated chicken $M x$ cDNA constructs within the pCI-neo vector and pEGFP-C3 vector, introducing a point mutation at 631 aa of the chicken Mx protein. Nucleotides of both plasmid DNAs corresponding to 631 aa were mutated by PCR by using mismatched primers, and the Ser-to-Asn mutated $M x$ cDNA construct in NG was designated as $\mathrm{NG}^{\mathrm{S} 631 \mathrm{~N}}$. Similarly, Asn-to-Ser mutated constructs in SHK and RI were referred as $\mathrm{SHK}^{\mathrm{N} 631 \mathrm{~S}}$ and $\mathrm{RI}^{\mathrm{N} 631 \mathrm{~S}}$, respectively. The following mismatched primers were $\begin{array}{llllll}\text { used } & \text { in these experiments: } & \text { NG } & \text { S631N } & \text { AM } & F\end{array}$ 
(5'-GATTGCTCAGGCGTTTACTTGCTCCAG-3') for the $\mathrm{NG}^{\mathrm{S} 631 \mathrm{~N}}$ construct, and SHK N631S AM F (5'-CTGGAGCAAGTAAACGCCTGAGCAATC-3') and R (5'-GATTGCTCAGGCGTTTACTTGCTCCAG-3') for the $\mathrm{RI}^{\mathrm{N} 631 \mathrm{~S}}$ and $\mathrm{SHK}^{\mathrm{N} 631 \mathrm{~S}}$ constructs. PCR was performed using Pfu DNA polymerase (Promega). The cycling profile comprised an initial denaturing step for $5 \mathrm{~min}$ at $95^{\circ} \mathrm{C}$, followed by 25 cycles at $95^{\circ} \mathrm{C}$ for $0.5 \mathrm{~min}, 55^{\circ} \mathrm{C}$ for $1 \mathrm{~min}, 72^{\circ} \mathrm{C}$ for $15 \mathrm{~min}$, and a final extension at $72^{\circ} \mathrm{C}$ for 15 min. PCR products were purified as described in section 2.3. Next, we confirmed the presence of mutations corresponding to 631 aa in all plasmids by nucleotide sequencing.

\subsection{Statistical analysis}

Statistical analysis of all data for comparison was carried out using the Fisher protected least significant difference (PLSD) test based on Statview (Abacus Concepts, Inc., Berkeley, CA). Experimental data are presented as means \pm standard errors. $P$ values of $<0.01$ were considered statistically significant. 


\section{Results}

\subsection{Sequencing analysis of chicken $M x c D N A$}

We sequenced chicken $M x$ cDNAs of 3 breeds of NG, RI, and SHK after cloning into the pGEM-T Easy Vector. When nucleotide sequences and predicted amino acid sequences of the 3 breeds were compared to those in the NCBI database (Genbank ID: NM 204609 and NP989940) from the WLR breed, 13 nucleotide substitutions were detected (data not shown), and 6 were accompanied by amino acid variations as shown in Table 1. Particularly, Mx protein of NG was estimated to have Ser at 631 aa, whereas those of both RI and SHK possessed Asn at 631 aa.

\subsection{Antiviral activity and intracellular localization of chicken Mx protein}

We prepared $M x$ cDNA constructs derived from NG, RI, and SHK within the pCI-neo vector and used an empty pCI-neo vector (pCI-neo) as a negative control. These 
constructs and pCI-neo were transfected into $3 \mathrm{~T} 3$ cells. $M x$ mRNA expression was confirmed using RT-PCR. Each cloned cell line transfected with the NG, RI, or SHK $M x$ cDNA construct expressed $M x$ mRNA, whereas expression was not observed in only the pCI-neo cell line (data not shown).

Infection experiments of $\mathrm{VSV} \Delta \mathrm{G}^{*}-\mathrm{G}$ into $3 \mathrm{~T} 3$ cells induces fluorescence from GFP integrated into the viral genome. Relative infectivity of $3 \mathrm{~T} 3$ cells with VSV $\Delta \mathrm{G}^{*}-\mathrm{G}$ was calculated and compared in to controls $3 \mathrm{~T} 3$ cells and the pCI-neo vector. As shown in Fig. 1A, cells expressing NG $M x$ mRNA showed 101.1\% relative infectivity. However, the relative infectivities of RI and SHK were suppressed to $31.5 \%$ and $28.5 \%$ respectively, indicating a significant decrease in the antiviral response $(P<0.01)$.

To examine the relationship between the antiviral activity against $\mathrm{VSV} \Delta \mathrm{G}^{*}-\mathrm{G}$ and intracellular localization of the chicken $\mathrm{Mx}$ protein, we attempted to observe localization using GFP-Mx-fused protein expression vectors. Constructs of NG, RI, and SHK $M x$ cDNAs within the pEGFP-C3 vector were prepared and transfected into mouse $3 \mathrm{~T} 3$ cells. Twenty-four hours after transfection, individual cells emanating GFP-fluorescent signals were observed (Fig. 1B). GFP signals were detected in the 
cytoplasm of 3T3 cells with respect to all breeds, but not in the nucleus. Surprisingly, we observed a characteristic difference in the fluorescent signals of the GFP-NG Mx-fused protein was homogenously distributed throughout the cytoplasm; however those of GFP-RI- and SHK-fused proteins showed granular-like distribution. According to these results, substitution of Ser and Asn at 631 aa of chicken Mx protein may be responsible for not only in vitro antiviral activity against VSV but also intracellular localization.

\subsection{Antiviral activity and intracellular localization of mutant chicken Mx protein}

To address whether the 631 aa substitution of chicken Mx protein is associated with not only antiviral activity but also intracellular localization, we used molecular mutants of recombinant vectors in which nucleotides coding for Ser and Asn at 631 aa were reciprocally altered. As shown in Fig. 2A, a nucleotide corresponding to Ser at 631 aa in the NG Mx protein was artificially replaced with Asn and designated as $\mathrm{NG}^{\mathrm{S} 631 \mathrm{~N}}$; in contrast, a nucleotide corresponding to Asn at 631 aa in the RI and SHK Mx proteins 
was converted to Ser, which was designated as $\mathrm{RI}^{\mathrm{N} 631 \mathrm{~S}}$ and $\mathrm{SHK}^{\mathrm{N} 631 \mathrm{~S}}$, respectively.

First, we determined the relative infectivity of cell lines expressing mutant chicken $M x$ mRNA (Fig. 2B). Cell lines transfected with $\mathrm{RI}^{\mathrm{N} 631 \mathrm{~S}}$ and $\mathrm{SHK}^{\mathrm{N} 631 \mathrm{~S}}$ constructs did not show clear antiviral activity against $\mathrm{VSV} \Delta \mathrm{G}^{*}-\mathrm{G}$; negative controls of $3 \mathrm{~T} 3$ and pCI-neo showed this pattern as well. The cell line transfected with the $\mathrm{NG}^{\mathrm{S} 631 \mathrm{~N}}$ construct showed a significantly higher antiviral response compared with negative controls $(P<0.01)$.

Moreover, we obtained the same positive results in an examination of intracellular localization using artificial mutants (Fig. 2C). In cell lines transfected with mutant $\mathrm{RI}^{\mathrm{N} 631 \mathrm{~S}}$ and $\mathrm{SHK}^{\mathrm{N} 631 \mathrm{~S}}$ constructs, fluorescent signals of GFP-Mx fused protein homogenously spread throughout the cytoplasm, similarly to the GFP-NG Mx-fused protein. In contrast, in a cell line transfected with the mutant $\mathrm{NG}^{\mathrm{S} 631 \mathrm{~N}}$ construct, fluorescent signals were distributed in a granular-like pattern. These results strongly indicate that the polymorphism at 631 aa of chicken Mx protein is correlated with both in vitro antiviral activity and intracellular localization. 


\section{Discussion}

We previously demonstrated that a polymorphism at 631 aa of the chicken Mx protein plays an important role in the antiviral activity against H5N1 influenza virus and VSV in vitro in cultured $3 \mathrm{~T} 3$ cells [12], and this has been verified by other groups. However, there is limited information regarding how the polymorphism at 631 aa of chicken $\mathrm{Mx}$ protein contributes to functional differences. Additionally, intracytoplasmic localization of human MxA protein and mouse MX1 is reportedly associated with both a conformational change of the protein and their antiviral activities $[4,14,16]$. If the polymorphism at the 631 aa of chicken Mx protein affects intracellular behavior, this difference may play a key role in its antiviral activity. In this study, we investigated the relationship between the antiviral activity and intracellular localization, focusing on the polymorphism of Ser or Asn at 631 aa of the chicken Mx protein.

We reconfirmed the nucleotide sequence of chicken $M x$ cDNA used in this study. NG was predicted to contain Ser at 631 aa, and RI and SHK contained Asn (Table 1). Moreover, 2 substitutions were detected in a significantly conserved domain, located at 
185 aa in SAS and at 632 aa in GED. The substitution at 185 aa has already been reported in a previous study [12], although this mutation was not found to affect antiviral activity. Another substitution at 632 aa was identified, and therefore, further experiments are required to clarify whether the substitution at 632 aa is associated with the antiviral activity.

We next measured antiviral activity and observed intracellular localization of chicken Mx protein (Fig. 1). NG Mx protein carrying Ser at 631 aa permitted VSV multiplication and homogenous spread of Mx throughout the cytoplasm. In contrast, in both RI and SHK, the Mx protein carrying Asn at 631 aa inhibited viral multiplication and was distributed in a granular-like pattern in the cytoplasm. These results indicated that a single amino acid substitution at 631 aa of chicken Mx protein affects both antiviral activity and intracellular localization.

As the final step, we constructed 3T3 cell lines expressing mutant chicken Mx protein in which there was a Ser-to-Asn or an Asn-to-Ser mutation at 631 aa. When antiviral activity and intracellular localization of mutant chicken Mx protein were examined, the results revealed that the mutant at 631 aa reciprocally altered their antiviral activities as 
well as their intracellular localization (Fig. 2). The genotype carrying Ser at 631 aa permitted viral multiplication and homogenous distribution in the cytoplasm. In contrast, the genotype carrying Asn at 631 aa was consistently shown to inhibit VSV multiplication and a granular-like Mx distribution pattern in the cytoplasm. Additionally, substitutions at 185 aa and 632 aa did not affect either antiviral activity or intracellular localization in $3 \mathrm{~T} 3$ cells. Thus, we concluded that a single amino acid substitution at 631 aa in the chicken $\mathrm{Mx}$ protein is responsible for both antiviral activity and intracellular localization.

In the human MxA protein, it has been postulated that GED binds to CID, mediating oligomerization and conformational change to target the viral structure [4]. However, it is unknown how this conformational change contributes to the antiviral function of the MxA protein. The information obtained in this study showed that chicken Mx carrying Asn at 631 aa distributes in a granular-like pattern in the cytoplasm, similar to that in human MxA. In contrast, in our thought, another chicken Mx carrying Ser at 631 aa existed as monomers in the cytoplasm. Human MxA protein reportedly interacts with ATP-dependent RNA helicase, DDX39A, and DDX39B proteins in the cytoplasm to 
inhibit viral replication [21]. In 3T3 cells, chicken $\mathrm{Mx}^{\mathrm{N} 631}$ proteins may interact with other protein such as ATP-dependent RNA helicases. However, in this study, we were unable to examine the oligomerization of chicken Mx protein and interaction with other proteins during viral invasion into the host cells. Further experiments are required to clarify whether the polymorphism at 631 aa of chicken Mx protein influences the antiviral activity through oligomerization and interaction with other proteins.

Several studies have presented conflicting information regarding whether the substitution at 631 aa of chicken Mx protein is important for antiviral activity. Some groups suggested that chicken $\mathrm{Mx}$ protein with Asn at 631 aa possesses higher pathogenic ability against H7N1 influenza virus in vivo compared to the Ser type [19], as well as exhibits higher inhibition of H5N2 influenza virus multiplication in vivo [3]. However, in contrast to our studies, several reports claimed that the polymorphism at 631 aa of chicken Mx protein does not influence antiviral activity $[1,17]$. However, in these studies, different cell types or different viral strains were used for in vivo and in vitro infectious experiments.

In conclusion, the present study demonstrates that the polymorphism at 631 aa in the 
chicken Mx protein play a significant role in both antiviral activity against VSV and intracellular localization in mouse $3 \mathrm{~T} 3$ cells. Future studies are necessary to analyze whether antiviral activity and intracellular localization of chicken Mx protein are closely related.

\section{Acknowledgments}

We thank Dr. Ayato Takada (Research Center for Zoonosis Control, Hokkaido

University, Japan) for kindly providing VSV $\Delta \mathrm{G}^{*}-\mathrm{G}$, and Dr. Makoto Takahashi (Field

Science Center for Northern Biosphere, Hokkaido University, Japan) for providing chicken blood. 


\section{References}

[1] C.T. Benfield, J.W. Lyall, G. Kochs, L.S. Tiley, Asparagine 631 variants of the chicken Mx protein do not inhibit influenza virus replication in primary chicken embryo fibroblasts or in vitro surrogate assays, J. Virol. 82 (2008) 7533-7539.

[2] D. Bernasconi, U. Schultz, P. Staeheli, The interferon-induced Mx protein of chickens lacks antiviral activity, J. Interferon Cytokine Res. 15 (1995) 47-53.

[3] S.J. Ewald, D.R. Kapczynski, E.J. Livant, D.L. Suarez, J. Ralph, S. McLeod, C. Miller, Association of Mx1 Asn631 variant alleles with reductions in morbidity, early mortality, viral shedding, and cytokine responses in chickens infected with a highly pathogenic avian influenza virus, Immunogenetics 63 (2011) 363-375.

[4] F. Flohr, S. Schneider-Schaulies, O. Haller, G. Kochs, The central interactive region of human MxA GTPase is involved in GTPase activation and interaction with viral target structures, FEBS Lett. 463 (1999) 24-28.

[5] M. Frese, G. Kochs, H. Feldmann, C. Hertkorn, O. Haller, Inhibition of bunyaviruses, phleboviruses, and hantaviruses by human MxA protein, J. Virol. 
70 (1996) 915-923.

[6] S. Gao, A. von der Malsburg, S. Paeschke, J. Behlke, O. Haller, G. Kochs, O. Daumke, Structural basis of oligomerization in the stalk region of dynamin-like MxA, Nature 465 (2010) 502-506.

[7] E. Gordien, O. Rosmorduc, C. Peltekian, F. Garreau, C. Brechot, D. Kremsdorf, Inhibition of hepatitis $\mathrm{B}$ virus replication by the interferon-inducible $\mathrm{MxA}$ protein, J. Virol. 75 (2001) 2684-2691.

[8] O. Haller, G. Kochs, Interferon-induced Mx proteins: dynamin-like GTPases with antiviral activity, Traffic 3 (2002) 710-717.

[9] H.K. Jin, T. Yamashita, K. Ochiai, O. Haller, T. Watanabe, Characterization and expression of the Mxl gene in wild mouse species, Biochem. Genet. 36 (1998) $311-322$.

[10] H.K. Jin, A. Takada, Y. Kon, O. Haller T. Watanabe, Identification of the murine Mx2 gene: Interferon-induced expression of the Mx2 protein from the feral mouse gene confers resistance to vesicular stomatitis virus, J. Virol. 73 (1999) 4925-4930. 
[11] M. Kanerva, K. Melen, A. Vaheri, I. Julkunen, Inhibition of puumala and tula hantaviruses in Vero cells by MxA protein, Virology 224 (1996) 55-62.

[12] J.H. Ko, H.K. Jin, A. Asano, A. Takada, A. Ninomiya, H. Kida, H. Hokiyama, M. Ohara, M. Tsuzuki, M. Nishibori, M. Mizutani, T. Watanabe, Polymorphisms and the differential antiviral activity of the chicken $M x$ gene, Genome Res. 12 (2002) 595-601.

[13] H. Landis, A. Simon-Jodicke, A. Kloti, C. Di Paolo, J.J. Schnorr, S. Schneider-Schaulies, H.P. Hefti, J. Pavlovic, Human MxA protein confers resistance to Semliki Forest virus and inhibits the amplification of a Semliki Forest virus-based replicon in the absence of viral structural proteins, J. Virol. 72 (1998) 1516-1522.

[14] M. Nakayama, K. Yazaki, A. Kusano, K. Nagata, N. Hanai, A. Ishihama, Structure of mouse Mx1 protein. Molecular assembly and GTP-dependent conformational change, J. Biol. Chem. 268 (1993) 15033-15038.

[15] S. Schneider-Schaulies, J. Schneider-Schaulies, A. Schuster, M. Bayer, J. Pavlovic, V. ter Meulen, Cell type-specific MxA-mediated inhibition of measles virus 
transcription in human brain cells, J. Virol. 68 (1994) 6910-6917.

[16] B. Schumacher, P. Staeheli, Domains mediating intramolecular folding and oligomerization of MxA GTPase, J. Biol. Chem. 273 (1998) 28365-28370.

[17] B. Schusser, A. Reuter, A. von der Malsburg, N. Penski, S. Weigend, B. Kaspers, P. Staeheli, S. Hartle, Mx is dispensable for interferon-mediated resistance of chicken cells against influenza A virus, J. Virol. 85 (2011) 8307-8315.

[18] M. Schwemmle, M.F. Richter, C. Herrmann, N. Nassar, P. Staeheli, Unexpected structural requirements for GTPase activity of the interferon-induced MxA protein, J. Biol. Chem. 270 (1995) 13518-13523.

[19] L. Sironi, J.L. Williams, A.M. Moreno-Martin, P. Ramelli, A. Stella, H. Jianlin, S. Weigend, G. Lombardi, P. Cordioli, P. Mariani, Susceptibility of different chicken lines to H7N1 highly pathogenic avian influenza virus and the role of Mx gene polymorphism coding amino acid position 631, Virology 380 (2008) $152-156$.

[20] A.Takada, C. Robison, H. Goto, A. Sanchez, K.G. Murti, M.A. Whitt, Y. Kawaoka, A system for functional analysis of Ebola virus glycoprotein, Proc. Natl. Acad. 
Sci. 94 (1997) 14764-14769.

[21] C. Wisskirchen, T.H. Ludersdorfer, D.A. Muller, E. Moritz, J. Pavlovic, Interferon-induced antiviral protein $\mathrm{MxA}$ interacts with the cellular RNA helicases UAP56 and URH49, J. Biol. Chem. 286 (2011) 34743-34751.

[22] H. Zhao, B.P. De, T. Das, A.K. Banerjee, Inhibition of human parainfluenza virus-3 replication by interferon and human MxA, Virology 220 (1996) 330-338.

[23] T. Zurcher, J. Pavlovic, P. Staeheli, Mouse Mx2 protein inhibits vesicular stomatitis virus but not influenza virus, Virology 187 (1992) 796-800.

[24] T. Zurcher, J. Pavlovic, P. Staeheli, Nuclear localization of mouse Mx1 protein is necessary for inhibition of influenza virus, J. Virol. 66 (1992) 5059-5066.

\section{Figure Legends}


Figure 1. Antiviral activity against $\mathrm{VSV} \Delta \mathrm{G}^{*}-\mathrm{G}$ and intracellular localization of chicken Mx protein. (a) Relative infectivities of VSV $\Delta \mathrm{G}^{*}-\mathrm{G}$ in $M x$-transfected mouse $3 \mathrm{~T} 3$ cells. Infectivity of non-transfected $3 \mathrm{~T} 3$ cells (3T3) is expressed as $100 \%$. The relative infectivity of each cell line was determined by dividing into the infectivity of 3T3. Shown are mean values \pm standard errors of the means. Significance levels at $P<0.01$ (*) compared with $3 \mathrm{~T} 3$ are indicated. (b) Intracellular localization of GFP-chicken Mx fused protein in mouse $3 \mathrm{~T} 3$ cells. A total of $300 \mathrm{ng} / \mathrm{mL}$ of several types of $\mathrm{NG}$, SHK, and RI $M x$ cDNA constructs within the pEGFP-C3 vector was transfected into mouse 3 T3 cells. After an additional 24-hours culture, transfected 3T3 cells were observed under a microscope $(\times 200)$, and the fluorescence of GFP, which was fused with chicken Mx protein, was detected at $470-490 \mathrm{~nm}$.

Figure 2. Antiviral activity against $\mathrm{VSV} \Delta \mathrm{G}^{*}-\mathrm{G}$ and intracellular localization of mutant chicken Mx protein. (a) Schematic representation showing construction of mutant chicken $M x$ cDNA. Mismatched primers were used in PCR, which was used to mutate 
the nucleotide corresponding 631 aa from Ser to Asn, or from Asn to Ser. (b) Relative infectivities of VSV $\Delta \mathrm{G}^{*} \mathrm{-G}$ in mutant $M x$-transfected mouse $3 \mathrm{~T} 3$ cells. Relative infectivities were the same as those shown in Fig. 1A. Shown are mean values \pm standard errors of the means. Significance levels at $P<0.01\left(^{*}\right)$ compared with 3T3 are indicated. (c) Intracellular localization of mutant GFP-chicken Mx-fused protein in mouse $3 \mathrm{~T} 3$ cells. The distribution of GFP-fluorescence for chicken Mx protein was same as shown in Fig. 1B. 
A

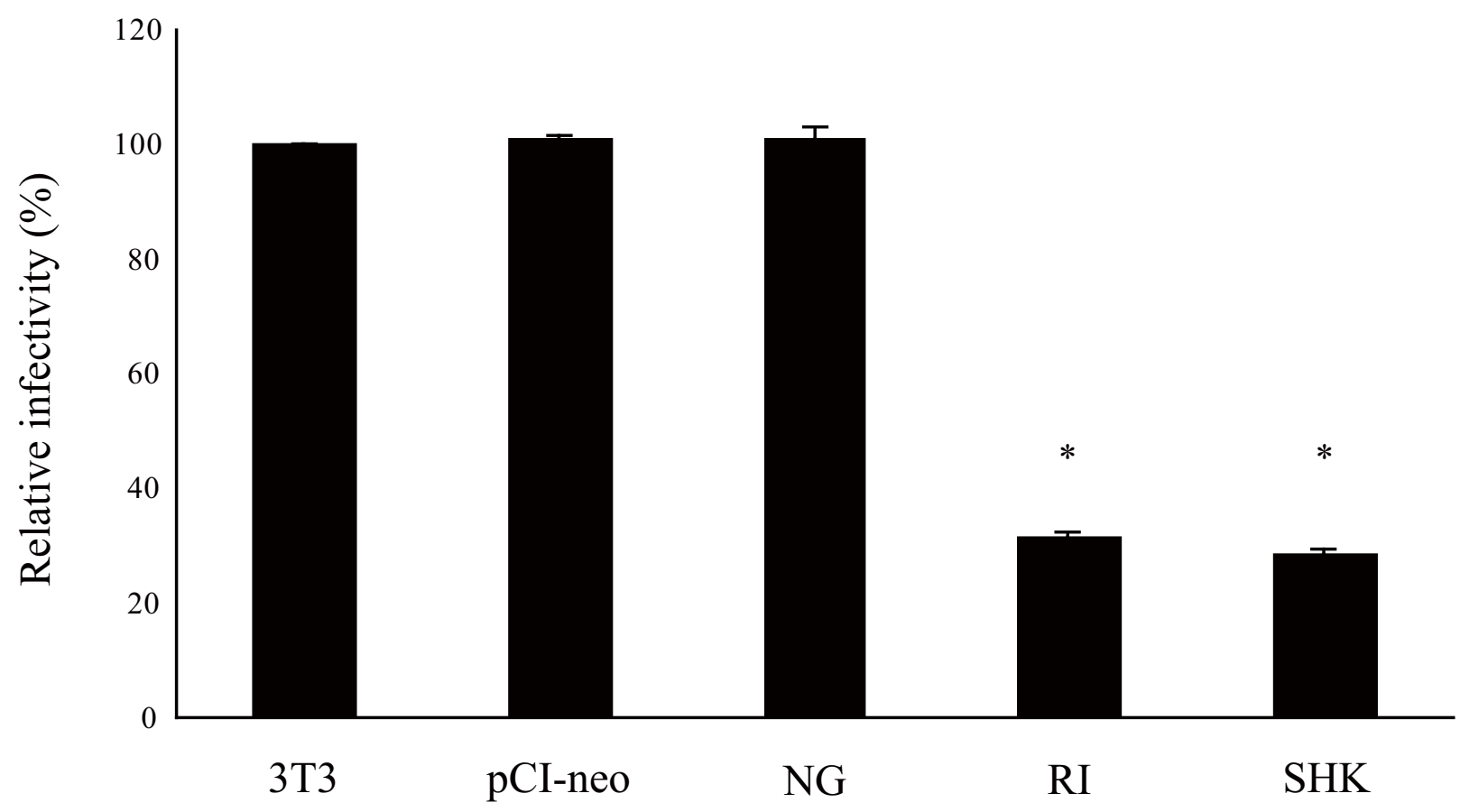

B

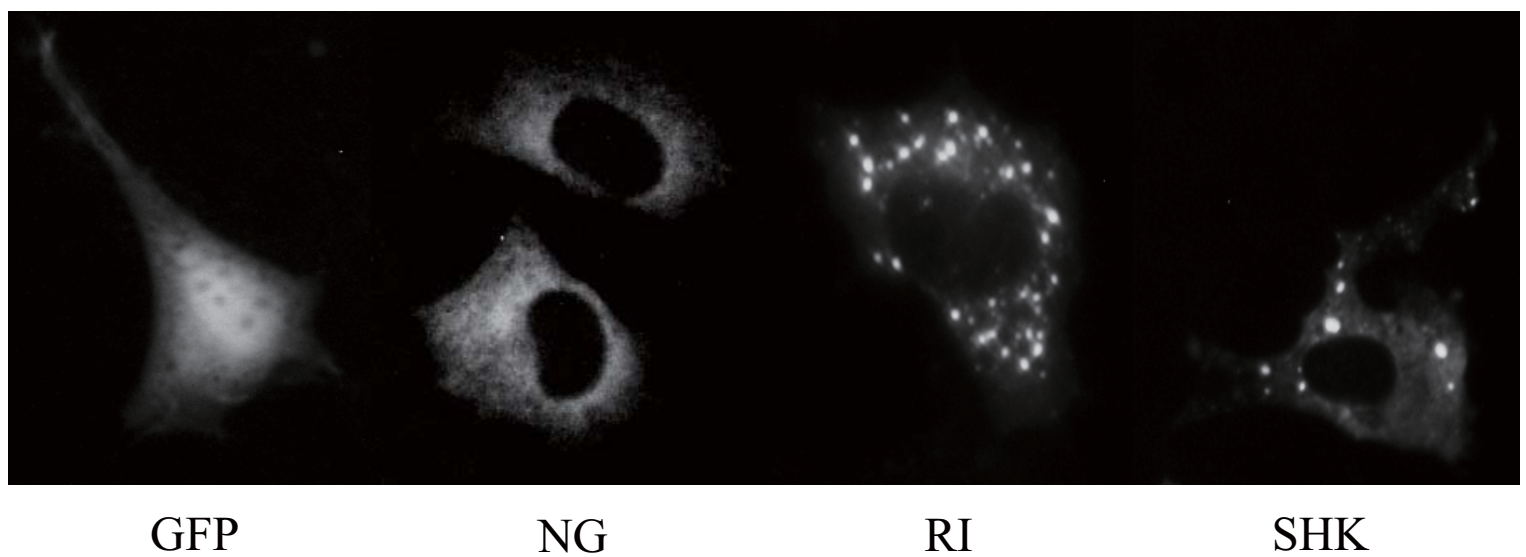

Figure 1 
A
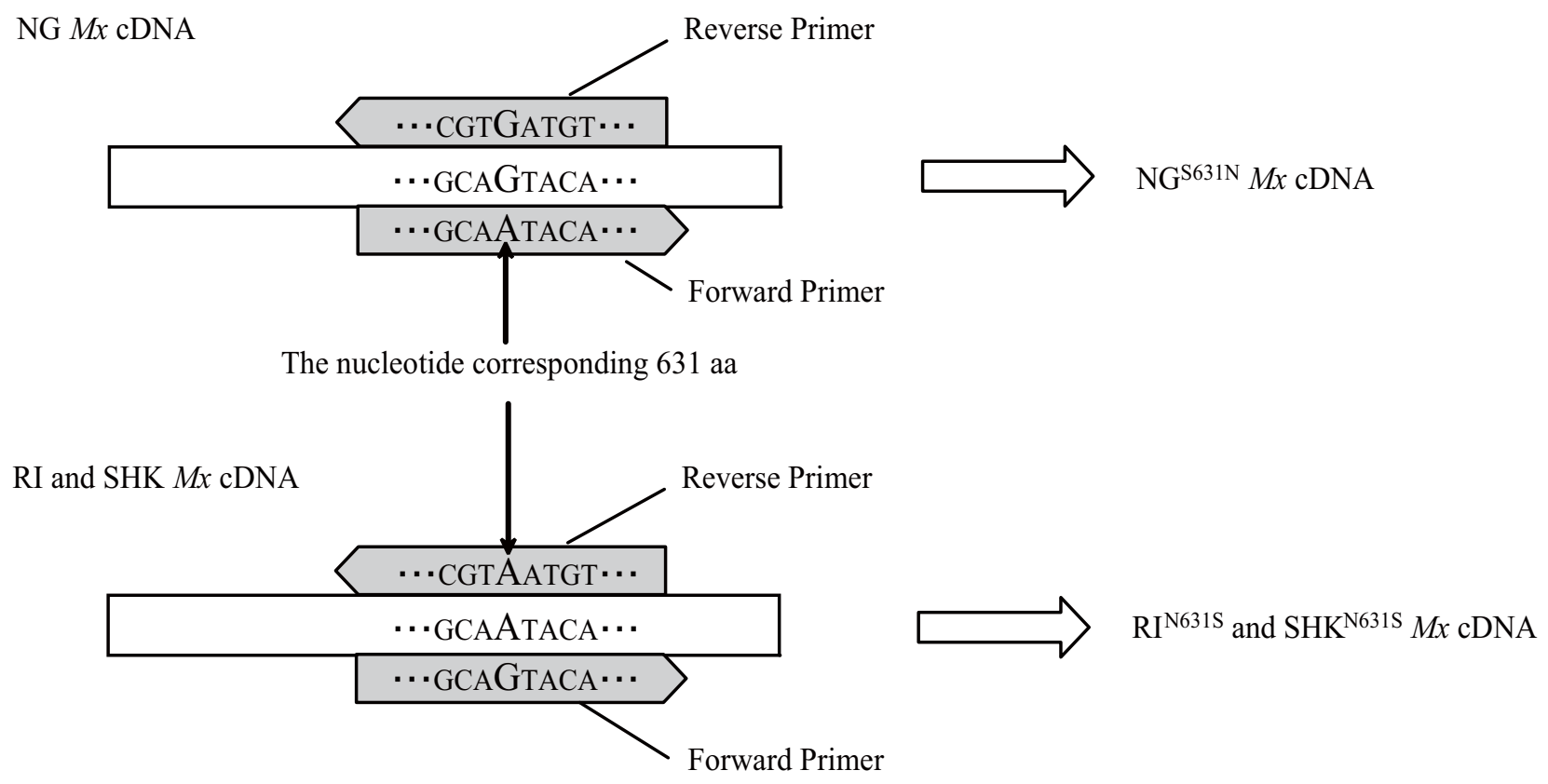

B

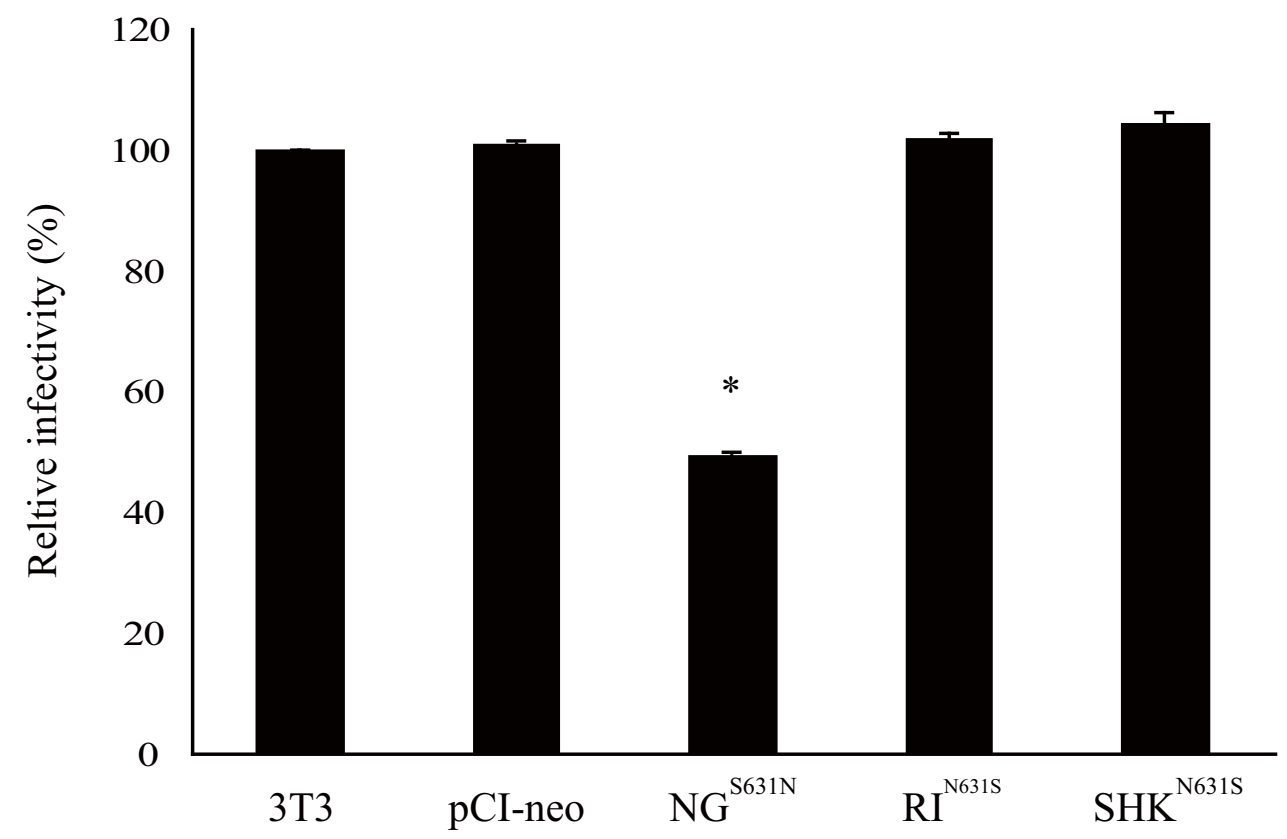

C

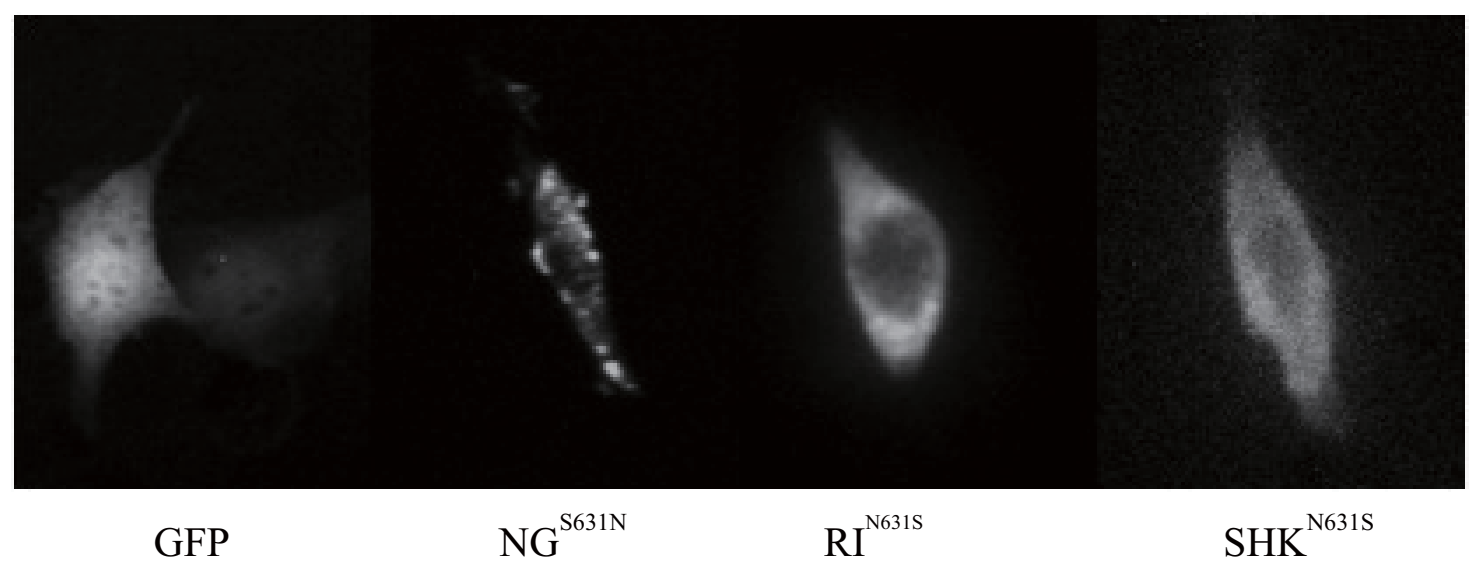

Figure 2 
Table 1. Nucleotide and predicted amino acid substitutions in the chicken Mx protein.

\begin{tabular}{lcccccc}
\hline & \multicolumn{5}{c}{ Amino acid position (nucleotide position) } \\
Breed & 72 & 85 & 185 & 198 & 631 & 632 \\
& $(230)$ & $(394)$ & $(694)$ & $(733)$ & $(2032)$ & $(2036)$ \\
\hline WLR & Asn & Leu & Lys & Asn & Ser & Lys \\
NG & Asn & Leu & Arg & Asn & Ser & Asn \\
SHK & Asn & Ser & Lys & Thr & Asn & Lys \\
RI & Asp & Leu & Lys & Thr & Asn & Lys \\
\hline
\end{tabular}

WLR, White Leghorn; NG, Nagoya; SHK, Shamo; RI, Rhode Island Red. 